\title{
Study on IR Bright Temperature with NOAA Data in Shanghai
}

\author{
Jun-Juan Zhao, Meng Yuan, \\ Yan-Li Guo, Cheng-Fan Li \\ School of Computer Engineering and Science, \\ Shanghai University, \\ Shanghai, China \\ E-mail: junjuanzhao@shu.edu.cn, 826907421@qq.com, \\ 464619548@qq.com, lchf@shu.edu.cn
}

\author{
Jing - Yuan Yin \\ Earthquake Administration of Shanghai Municipality, \\ Shanghai, China \\ E-mail: 1061233142@qq.com
}

\begin{abstract}
Infrared brightness temperature is a key index in the field of seismic precursory. However, it isn't received the attention of the professional field because of the limitations of data sources. With the development of remote sensing technology, more and more thermal infrared remote sensing data is obtained. In the paper, in order to study a new method of seismic precursory, we use AVHRR from NOAA satellite to compute Ten-day background field of brightness temperature in Shanghai area
\end{abstract}

Keywords-remote sensing; NOAA; thermal infrared brightness temperature; seismic precursory

\section{INTRODUCTION}

Shanghai is a modern international metropolis located on the coast of the East China Sea, with a developed economy, a high population density, a high level of urbanization and fast development. In the event of an earthquake and tsunami caused by the earthquake, people's lives and property and economic losses will be immeasurable. Therefore, to strengthen and enhance the earthquake monitoring and forecasting work in Shanghai not only has important significance of scientific research, but also the urgent needs of earthquake disaster reduction in Shanghai. However, due to the special geographical position of Shanghai, one side is the real estate, factories, densely populated, high cost of land in the metropolis, while the soft soil of coastal areas, which makes the traditional ground seismic monitoring station construction is very difficult. On the one hand, it is difficult to find a suitable place to build sites, on the other hand a great impact on urban interference is not conducive to the capture of seismic signals. Over the years a large number of observations show that in the process of earthquake with the ground temperature, temperature anomalies such as abnormal temperature anomalies. Therefore, it is very important and necessary to apply satellite thermal infrared remote sensing based on space-to-earth observation to the prediction of earthquake monitoring in Shanghai, and to give full play to the advantages of space observation technology and supplement the limited ground observation.

\section{DATA SOURCES}

Currently, more satellite data are available for research. There are now NOAA Class $1 \mathrm{~B}$ data, MODIS Class 1B data, MODIS Level 2 surface temperature products, and FY Class 1B data. FY data is currently low resolution, and previously received data is FY-2C, FY-2E is now receiving the data domestic. MODIS received $1 \mathrm{~B}$ data is bad quality, but MODIS 2-level products, including land surface temperature, is easy to access and free. So this selection of infrared brightness temperature data is mainly used to determine the temperature of the surface temperature from NOAA data simultaneously with MODIS 2B data.

\section{DATA PROCESSING}

\section{A. Bright temperature Data Preprocessing}

1) Geometric correction: Compared with the target brightness temperature, the data obtained by infrared remote sensing technology are distorted due to the physical characteristics of the sensor object, the solar azimuth and the atmospheric effect. Therefore, the data need to be pretreated to eliminate or correct the various noise in the process of remote sensing image formation. The data used in this paper mainly geometric correction using the ground control point to meet the research needs.

The geo-localization file that comes with the satellite sensor can aid in geometric correction when calibrating with a ground control point. First determine the reference map, in the two images by selecting the control point match another grid file, so that the same surface objects appear in the corrected image the same location. In the control point, each scan line has 51 positioning data, that is, the $\mathrm{X}$ direction of the correction data should be less than or equal to $51, \mathrm{Y}$ direction of the correction data should be less than or equal to the number of rows, the data can be used to provide latitude and longitude .

2) Cloud removal and denoising: Clouds and noise are the most disturbing effects of satellite reception. Surface radiation is blocked by clouds and cannot be received by sensors. The noise is caused by complicated factors, which cause some ineffective information during satellite reception to reduce the image quality. Therefore, it is necessary to remove the cloud image and denoise the satellite image, so as to establish a normal and accurate regional brightness temperature background field. In this paper, the target data are processed according to the specific processing method proposed by Wen Shaoyan. Firstly, the cloud and noise of 
the target area are analyzed statistically, and the brightness temperature thresholds are determined respectively. Then, the thresholds are designed based on the thresholds based Cloud and Noise Detection Model. The threshold value of the indicator is calculated by adding and subtracting the bright temperature average value and the standard temperature difference. According to previous studies, it is known that the brightness temperature of the general cloud is lower than the surface brightness temperature, and the brightness temperature of the noise is much higher than the surface brightness temperature.

\section{B. Bright Temperature Calculation}

The brightness temperature data are obtained by satellite remote sensing image, which is a typical surface data with wide coverage and large amount of information. The surface temperature data are used to characterize the land surface temperature trough some calculation method. And establishing the normal background field in the region, it has great significance to the future of thermal anomaly monitoring.

1) The principle of infrared light temperature calculation: The actual observed radiation value of a satellite radiometer, using the Planck function and the band response function is converted into a blackbody temperature, the blackbody temperature is the brightness temperature. It should be noted that the radiation value detected here is a band of radiation within the integrated value, so it is to take into account not only the response of the detector function, but also consider what the sensor is located on the platform. Therefore, the first need to detect the radiation value of the satellite calibration processing, and then converted into the corresponding brightness temperature value. The key point of the brightness temperature is to assume that the measured radiation value is the result of the black body surface radiation, so the radiation value measured by the direction of $(\theta, \varphi)$, and $R_{i}(\theta, \varphi)$ (radiation value of the i-th band) and the brightness temperature of the target $\left(T_{i}(\theta, \varphi)\right)$ satisfy the following relation:

$$
R_{i}(\theta, \varphi)=R_{i}\left[T_{i}(\theta, \varphi)\right]=\int_{\lambda_{1}}^{\lambda_{2}} \frac{f_{i}(\lambda) C_{1}}{\pi \lambda^{5}\left[\exp \left(\frac{C_{2}}{\lambda T_{i}(\theta, \varphi)}\right)-1\right]} d \lambda
$$

Where in, $\lambda$ is wavelength, $\lambda_{1}$ is lower limit and $\lambda_{2}$ is upper limit of the i-th band. $C_{1}=3.7404 \times 10^{8} \mathrm{~W} \cdot \mu \mathrm{m}^{4} \cdot \mathrm{m}^{-2}, C_{2}=14.387 \times 10^{-3} \mathrm{~K}$, $f_{i}(\lambda)$ is the sensor band response function of the i-th band, it can be normalized by the following equation:

$$
\int_{\lambda_{1}}^{\lambda_{2}} f_{i}(\lambda) d \lambda=1
$$

The target brightness temperature can calculate through the inverse function of the type.
2) The method of establishing the background field of infrared light temperature: After preprocessing the infrared brightness temperature data, the infrared brightness temperature data are calculated by the formula (2). Then the mean and standard deviation of the brightness temperature data can be used to construct the characterization of the background field in chronological order. The specific calculation method is as follows:

The thermal infrared brightness temperature anomaly information before earthquake is relative to the normal dynamic background field. In order to extract the anomaly information of thermal infrared before the earthquake, the normal brightness background field must be set up, which will be used as the judgment index.

Let $\mathrm{N}$ be the available data age, $\mathrm{Ti}(\mathrm{x}, \mathrm{y}, \mathrm{t})$ is the observation value of the brightness temperature at the time point $t$ of the i-th year at the observation position (x, y), T (x, $y, t)$ indicates average brightness temperature and $\sigma(x, y, t)$ is mean standard deviation of the observation area at the $t$ time within $\mathrm{N}$ years, when there isn't any seismic activities, $\mathrm{T}(\mathrm{x}, \mathrm{y}, \mathrm{t})$ and $\sigma(\mathrm{x}, \mathrm{y}, \mathrm{t})$ can be solved using the following equation:

$$
\begin{aligned}
& \mathrm{T}(\mathrm{x}, \mathrm{y}, \mathrm{t})=\frac{1}{N} \sum_{i=0}^{N-1} T_{i}(\mathrm{x}, \mathrm{y}, \mathrm{t}) \\
& \sigma(\mathrm{x}, \mathrm{y}, \mathrm{t})=\sqrt{\frac{1}{N-1} \sum_{i=0}^{N-1}\left(T_{i}(\mathrm{x}, \mathrm{y}, \mathrm{t})-\mathrm{T}(\mathrm{x}, \mathrm{y}, \mathrm{t})\right)^{2}}
\end{aligned}
$$

\section{BRIGHT TEMPERATURE RESEARCH IN SHANGHAI}

\section{A. Overview of the Study Area}

Shanghai and adjacent areas are located in the eastern of China, across the North Korea block, the Yangtze fault block and the Qinling-Dabie fault in the tectonic position and east of South Yellow Sea, where the geological structure is very complex. Therefore, it is a basic task to carry out earthquake prediction and prevention by monitoring the earthquake precursory movement through various advanced technical means and sketching out the pre-earthquake monitoring network in this area.

In this paper, the Yangtze River estuary earthquake monitoring circle must be included within the scope of the study area. From the multi-research point of view, the scope of specific observational area needs to meet the following several elements:

- It shall include the Yangtze River Estuary Earthquake Monitoring Circle (119.6 123.6 $\mathrm{E}$, $30 \sim 33^{\circ} \mathrm{N}$ );

- It must include the South Yellow Sea multiple earthquake areas;

- The general study area is $10^{\circ} \times 10^{\circ}$, which can be used as a reference;

- It should be included as much as possible different types of geological structure. 


\section{B. Bright Temperature Background Field in Shanghai}

1) Bright teperature database:Bright temperature is obtained from NOAA, FY and MODIS satellite. NOAA / AVHRR Polar-Orbiting Meteorological Satellite Receiving System receives NOAA 15,16,17,18 (orbit)and 19 (orbit) .China's meteorological satellite FY-3A, 3B, 3C satellite data which was successfully built and put into use in June 2003 by The Institute of Geology of China Earthquake Administration .Satellite data is received every 4-6 hours , about 12 stripe images per day. The MODIS data can be obtained through the official website free of charge.

The data are processed in batches of standard atmospheric correction, radiation correction, cloud detection, area cutting and projection and coordinate transformation. The final image database includes 2677 images with a capacity of 34.8GB, which is mainly from NOAA / AVHRR satellite thermal-infrared light-temperature image database (Table1,Table2) of Shanghai and surrounding areas (114 ${ }^{\circ}$ $126^{\circ} \mathrm{E}, 27^{\circ}-35^{\circ} \mathrm{N}$ ) from 2010 to 2015.

2) Establishment of infrared bright temperature background and analysis of space - time evolution:Satellite infrared brightness temperature is a comprehensive reflection of thermal radiation, affected by the surface and the atmosphere and other factors of especially the impact of solar radiation. In the establishment of the Shanghai infrared brightness temperature background field, this paper selects NOAA / AVHRR polar metric satellite images at night in Shanghai and adjacent areas $\left(116^{\circ}-126^{\circ} \mathrm{N}, 27^{\circ}-35^{\circ} \mathrm{N}\right)$ in 2010-2016 to avoid the sun radiation effects, while for some missing data, we use MODIS data for correction. In this study, the background temperature field of the infrared temperature field is studied systematically, and the temporal and spatial evolution characteristics and influencing factors of the bright temperature background field are analyzed in the study area of Shanghai.

\section{DISCUSSION AND CONCLUSION}

The statistics of the background field of the study area are obtained by dividing a month into three periods, and the background field value is calculated, which reflects the short-term change of brightness temperature. From the time series, the time variation of bright temperature in Shanghai is closely related to the seasonal alternation: Such as the spring and summer alternation of April, May and autumn and winter alternation of October, November cooling background temperature field has obvious discrepancy of variations. Specific look in May of at the turn of spring and summer, the background temperature field of the upper, middle and the end was gradually increased, the bright temperature of the background field decreased gradually in October, but the background temperature of the other months did not change obviously. Spatial distribution of the brightness temperature changes are mainly affected by rivers, water bodies, topographic factors and fault structural belt, etc., the temperature at the fault zone is higher than the background around. In summary, the phenomenon of bright temperature and season is closely related to the short time scale of one month, which shows that it has strong spatial correlation.

\section{REFERENCES}

[1] WEN Shao-yan,QU Chun-yan,YAN Li-li, et al. Method for Constructing Regional Brightness Temperature Background Field and its Preliminary Application. Earthquake, 2011, vol31 (2):59-67

[2] Auroop R.Ganguly, Karsten Steinhaeuser. Data Mining for Climate Change and Impacts Proceedings of the 2008 IEEE International Conference on Data Mining.Wokshops Dec.2008, 150-155

[3] Aurelie C.Lozano, Hongfei Li, et al. Spatial-temporal causal modeling for climate change attribution Knowledge discovery and data mining Jul.2009,Paris,France

[4] ZHANG,YuanSheng,Xiao,ZHONG,MeiJiao.Wenchuan earthquake: Brightness temperature changes from satellite infrared information.Chinese Science Bulletin, 2010, 55(18):1917-1924

[5] Q Meng,X Lu,N Shao,X Shen,F Geng. Research progress in earthquake infrared background field and brightness temperature background field foundation of the demonstration areas. Acta Seismologica Sinica, 2016,57-64

[6] Zhang X, Zhang Y S, Wei C X, et al. Analysis of Thermal Infrared Anomaly before the Lushan M_S7.0 Earthquake[J]. China Earthquake Engineering Journal, 2013,23-30 
TABLE 1. The number of bright temperature image database details (unit: scene)

\begin{tabular}{|c|c|c|c|c|c|c|c|c|c|c|c|c|}
\hline & Jan. & Feb. & Mar. & Apr. & May & Jun. & Jul. & Aug. & Sep. & Oct. & Nov. & Dec. \\
\hline 2010 & 29 & 28 & 31 & 30 & 30 & 31 & 32 & 35 & 31 & 34 & 33 & 36 \\
\hline 2011 & 36 & 33 & 36 & 36 & 39 & 36 & 38 & 41 & 39 & 40 & 40 & 44 \\
\hline 2012 & 43 & 41 & 48 & 44 & 45 & 47 & 49 & 49 & 49 & 51 & 52 & 56 \\
\hline 2013 & 53 & 50 & 56 & 54 & 55 & 51 & 46 & 50 & 46 & 50 & 44 & 43 \\
\hline 2014 & 44 & 37 & 40 & 41 & 37 & 37 & 36 & 35 & 33 & 32 & 31 & 29 \\
\hline 2015 & 46 & 39 & 42 & 43 & 39 & 40 & 37 & 36 & 34 & 35 & 31 & 31 \\
\hline
\end{tabular}

TABLE 2. Brightness temperature image database capacity details (unit: MB)

\begin{tabular}{|l|c|c|c|c|c|c|c|c|c|c|c|c|}
\hline & Jan. & Feb. & Mar. & Apr. & May & Jun. & Jul. & Aug. & Sep. & Oct. & Nov. & Dec. \\
\hline 2010 & 386 & 373 & 413 & 399 & 399 & 413 & 426 & 466 & 413 & 453 & 439 & 479 \\
\hline 2011 & 479 & 439 & 479 & 479 & 519 & 479 & 506 & 546 & 519 & 532 & 532 & 586 \\
\hline 2012 & 572 & 546 & 639 & 586 & 599 & 626 & 652 & 652 & 652 & 679 & 692 & 745 \\
\hline 2013 & 706 & 666 & 745 & 719 & 732 & 679 & 612 & 666 & 612 & 666 & 586 & 572 \\
\hline 2014 & 586 & 493 & 532 & 546 & 493 & 493 & 479 & 466 & 439 & 426 & 413 & 386 \\
\hline 2015 & 612 & 519 & 559 & 572 & 519 & 532 & 493 & 479 & 453 & 466 & 413 & 413 \\
\hline
\end{tabular}

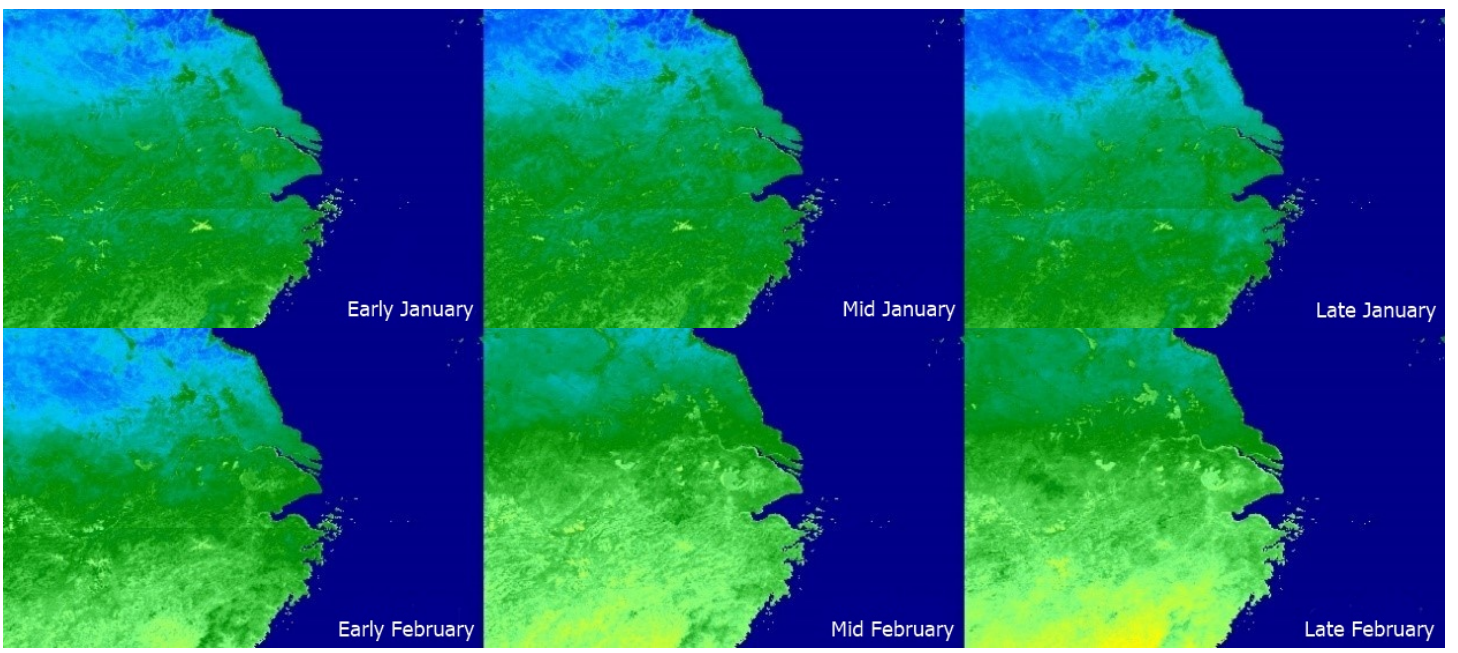

(a) 


\section{ATLANTIS
PRESS}

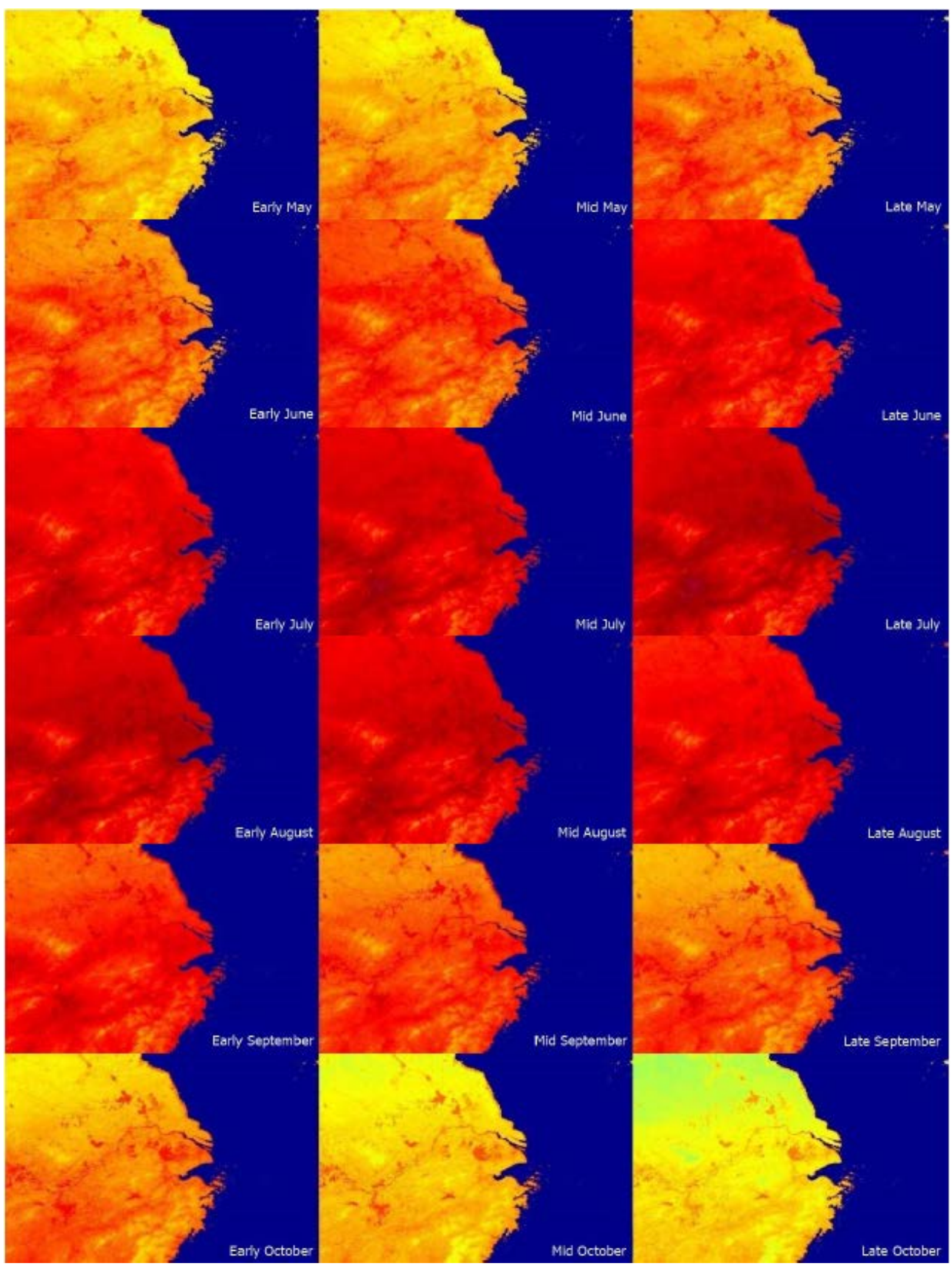

(b) 


\section{ATLANTIS
PRESS}
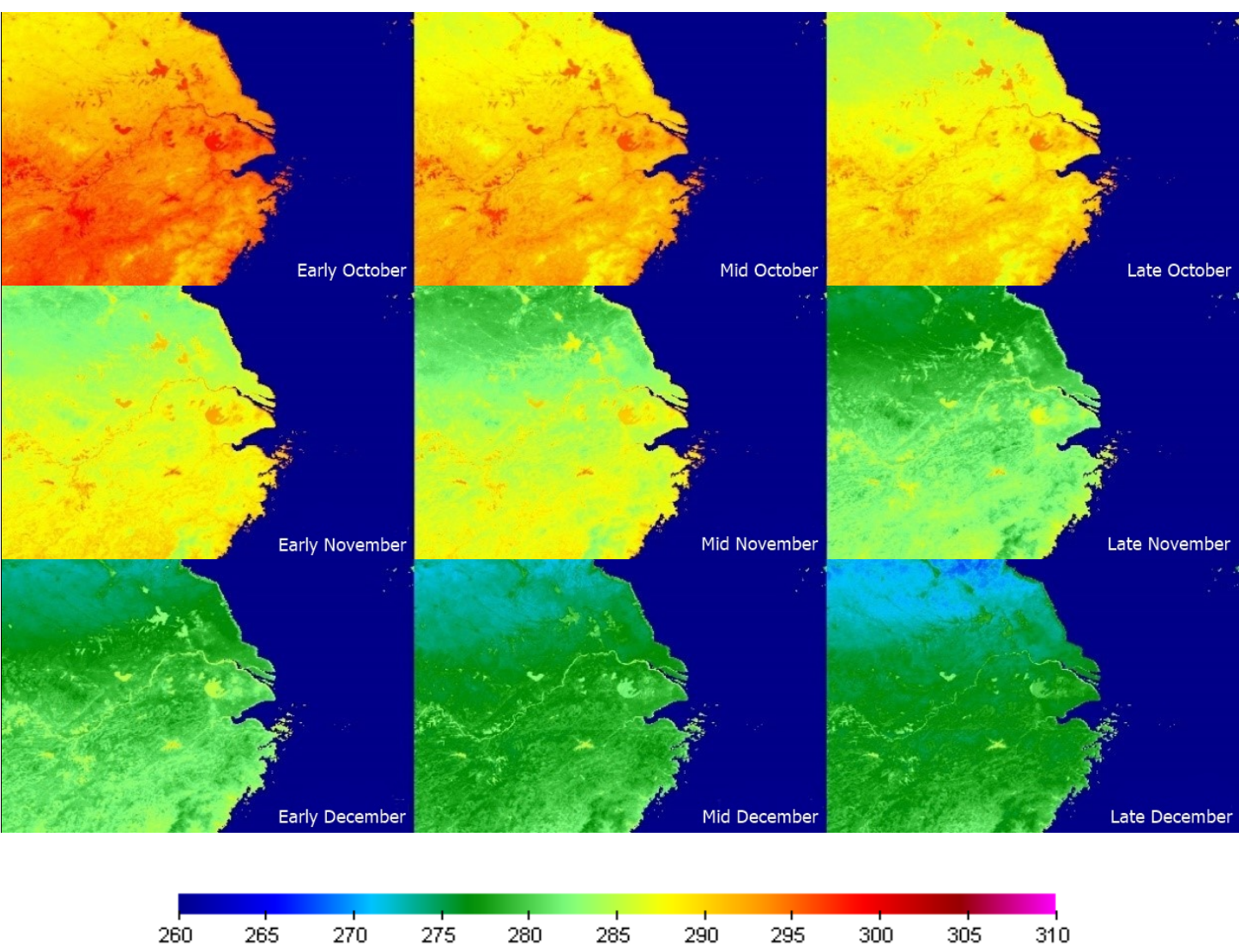

(c)

Figure 1. Shanghai Research Area for many years the temperature of the background field 